\title{
Modelado actual y futuro de LA IDONEIDAD DE HÁBITAT El AHUEHUETE (TAXODIUM MUCRONATUM TEN.): UNA PROPUESTA PARA CONSERVACIÓN EN MÉXICO

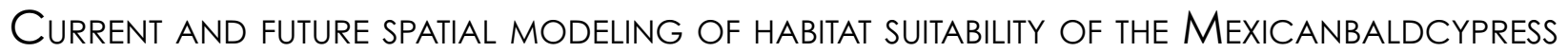 (TAXODIUM MUCRONATUM TEN.): a PROPOSAL FOR CONSERVATION IN MEXICO
}

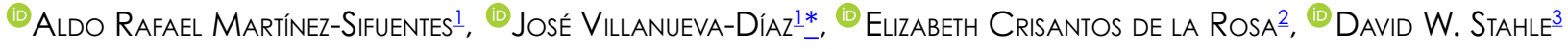

\author{
1 Instituto Nacional de Investigaciones Forestales, Agrícolas y Pecuarias, Centro Nacional de Investigación Disciplinaria en \\ Relación Agua, Suelo, Planta y Atmósfera, Gómez Palacio, Durango, México. \\ ${ }^{2}$ Universidad Autónoma Agraria Antonio Narro, Unidad Laguna, Torreón, Coahuila, México. \\ ${ }^{3}$ Universidad de Arkansas, Departamento de Geociencias, Fayetteville, Arkansas, EUA. \\ *Autor para la correspondencia: jvilladi@prodigy.net.mx
}

\section{Resumen}

Antecedentes: Los modelos para determinar la idoneidad de hábitat de especies arbóreas es actualmente una herramienta geoespacial relevante en la toma de decisiones.

Hipótesis: La idoneidad de hábitat actual y futura permite identificar zonas potenciales para conservación del ahuehuete en México.

Especie de estudio: Taxodium mucronatum Ten.

Sitio de estudio: México.

Métodos: Se emplearon registros de ahuehuete y variables climáticas, edafológicas y topográficas, las cuales se procesaron a través del algoritmo MaxEnt; se estimaron superficies actuales y futuras para los escenarios 2050, 2070 con dos criterios de distribución, idoneidad de hábitat y ecosistema actual o ripario y se identificaron áreas potenciales para conservación.

Resultados: Se estimó una superficie de 11,217,706.2 y 48,958.5 ha como áreas de idoneidad de hábitat, bajo los dos criterios, respectivamente. La variable más importante en el modelo fue la temperatura promedio del trimestre más frío. El modelo predice una reducción en superficie potencial de 2,989,580.9 ha y de 7,158,9829.96 ha para 2050 y 2070, respectivamente. La reducción estimada en la superficie actual es de $12,415.3$ ha y 32,037.1 ha, para 2050 y 2070, respectivamente. Se identificaron diversas zonas para conservación en los estados de Puebla, Guanajuato, Estado de México y Chiapas.

Conclusiones: En el presente estudio, se generó una propuesta para selección de sitios y desarrollo de actividades de conservación y restauración del ahuehuete en México, mediante el algoritmo MaxEnt.

Palabras claves: Cambio climático, escenarios climáticos, idoneidad de hábitat.

\section{Abstract}

Background: Models to determine the habitat suitability of species is becoming important as a relevant geospatial tool in decision-making. Hypothesis: Modeling of current and future habitat suitability of Mexican baldcypress allows identifying potential conservation areas in Mexico. Study species: Taxodium mucronatum Ten.

Study site: Mexico.

Methods: Distribution records of Mexican baldcypress and climatic, edaphological and topographic variables were processed through the MaxEnt algorithm from where current and future areas for two distribution criteria, habitat suitability and current or riparian ecosystem were estimated, and potential conservation areas identified.

Results: An area of 11,217,706.2 and 48,958.5 ha was estimated as potential and current habitat suitability, for two criteria for the Mexican baldcypress, respectively. The most important variable in the model was the average temperature of the coldest quarter. The model for the 2060 and 2070 scenarios forecasted a reduction of 2,989,580.9 ha, and 7,158,929.9 ha, respectively, and a reduction in current area of 12,415.3 ha, and 32,037.1 ha, respectively; sites for conservation were identified in the states of Puebla, Mexico, Guanajuato, and Chiapas.

Conclusions: We presented a proposal for conservation and restoration activities of Mexican baldcypress based on the MaxEnt algorithm. Key words: Climate change, climatic scenarios, habitat suitability.

Este artículo se encuentra bajo los términos de la licencia Creative Commons Attribution License CCBY-NC (4.0) internacional. https://creativecommons.org/licenses/by-nc/4.0/ 
Los sistemas forestales son hábitat de una amplia diversidad biológica, los cuales, proporcionan una serie de servicios ecológicos, económicos y sociales, entre los que se encuentran la regulación del ciclo hidrológico, captura de carbono y belleza escénica (CONAFOR 2009). Los sistemas arbóreos que se desarrollan a orillas de arroyos o ríos, forman un tipo de vegetación conocida como bosque de galería, vegetación riparia o vegetación ribereña (Naiman et al. 1993). La vegetación disminuye la erosión del suelo, favorece la filtración de nutrientes y contaminantes, mejorar la calidad del agua e incrementa la recarga del acuífero (Hession et al. 2000). Aunado a los beneficios ecológicos que proporciona el ecosistema ripario, este tipo de vegetación es de gran belleza escénica y da lugar a sitios recreativos que podrían ser utilizados con fines ecoturísticos (Enríquez-Peña \& Suzán-Azpiri 2011).

La familia Cupreassaceae es un grupo de coníferas cosmopolitas, localizados tanto en climas cálidos como templados-frescos (Carranza 1992). Una especie característica de la familia es, el ahuehuete o sabino. Existe debate en cuanto a su clasificación taxonómica, ya que Denny \& Arnold (2007) lo clasifican como Taxodium distichum var. mexicanum (Carriére) Gordon \& Glend., sin embargo en este estudio, lo consideramos como tradicionalmente se clasifica en la literatura para México, es decir, Taxodium mucronatum Ten. (Villaseñor 2016). El ahuehuete, es un árbol de apariencia majestuosa que alcanza una altura de hasta $40 \mathrm{~m}$, corteza color café claro, ramillas colgantes con hojas casi rectas de 8 a $200 \mathrm{~mm}$ de largo, con inflorescencia masculina de 15 a $30 \mathrm{~cm}$ de largo, conos globulosos a ovales, color verde con escamas arrugadas (Martínez 1963). Algunos sinónimos del ahuehuete son Taxodium huegelii Lawson, Taxodium montezumae Decaisne, Taxodium mexicanum Carriere y Taxodium virens Beissn. El ahuehuete es una especie de hábitat ripario, con grandes requerimientos de agua, ubicándose en corrientes permanentes o semipermanentes y ocasionalmente en sitios sin contacto directo con el agua, pero con un manto freático superficial, donde tiene acceso directo al recurso hídrico (Villanueva Díaz et al. 2003).

Se ha determinado que diferentes actividades humanas impactan de manera significativa los ecosistemas riparios de México. El régimen hidrológico es alterado por la extracción de material pétreo, la desviación de volúmenes de agua, la explotación ganadera en los márgenes de ríos, incendios intencionales y daños directos al arbolado por actos vandálicos, entre otros disturbios antropogénicos. El uso intensivo del agua en cauces de ríos, donde se ubica la especie, aunado a problemas de contaminación y ocu- rrencia de eventos extremos de sequía han diezmado notablemente las poblaciones del ahuehuete, situación que demanda acciones urgentes de preservación y de restauración (Villanueva Díaz et al. 2007).

Modelos predictivos del clima fundamentados en gases efecto invernadero, señalan un aumento en la temperatura media anual de hasta $2{ }^{\circ} \mathrm{C}$ para 2050 , con efectos significativos en la biodiversidad mundial (Abram et al. 2019). Ante tales modificaciones en el clima, la biodiversidad actual tendría tres posibles alternativas de sobrevivencia, tolerar las alteraciones climáticas, la extinción local y/o regional, o cambiar sus áreas de distribución (Cruz-Cárdenas et al. 2016). La vegetación ribereña podría tener un mayor impacto ante el cambio climático con repercusiones en su distribución actual, debido a las restricciones de adaptación que se prevén ocasionadas por la modificación de clima (Davis \& Shaw 2001).

El conocimiento de la idoneidad de hábitat de especies forestales, permite distinguir los diferentes patrones ambientales que contribuyen en su establecimiento, lo que favorece la obtención de información importante en actividades de conservación y gestión de recursos genéticos (Hernández-Ruíz et al. 2016). Actualmente, se cuenta con la disponibilidad de herramientas que ayudan a generar escenarios de distribución de especies (Elith et al. 2006). Dichas herramientas consisten en representaciones cartográficas, donde la distribución espacial de una especie es geográficamente explícita, en función a un grupo de variables de carácter continuo o categórico de naturaleza climática, edafológica y topográfica (Guisan \& Zimmermann 2000). El programa MaxEnt es un algoritmo especializado que se ha usado con frecuencia para predecir la idoneidad de hábitat actual y futura de coníferas en México (Manzanilla-Quiñones et al. 2019a). Este programa emplea registros de presenciaausencia, ha sido ampliamente utilizado para estimar la idoneidad de hábitat de especies de diversos grupos biológicos (Phillips et al. 2006), que han resultado útiles, sobre todo para la elaboración de programas enfocados a conservación y/o restauración ecológica (Sáenz-Romero et al. 2015, Hu et al. 2020, Martínez-Sifuentes et al. 2020).

En este trabajo se plantearon los siguientes objetivos: i) delimitar y estimar las áreas de idoneidad de hábitat del ahuehuete y de la vegetación riparia; ii) identificar las variables ambientales relevantes con base a su idoneidad de hábitat actual y futuro de la especie (2041-2060, 20612080) y iii) proponer áreas de conservación de la especie para México. 
Distribución actual y futura de Taxodium mucronatum Ten.

\section{Materiales y métodos}

Área de estudio. El presente estudio se circunscribe al área con presencia del ahuehuete en México, delimitada por las coordenadas extremas $16^{\circ} 0^{\prime} 00^{\prime}$ a $28^{\circ} 30^{\prime} 00^{\prime \prime}$ de latitud norte, y $92^{\circ} 0^{\prime} 00^{\prime}$ a $109^{\circ} 30^{\prime} 00^{\prime}$ de longitud oeste, con una superficie aproximada de $1,059,155.81 \mathrm{~km}^{2}$ y con un gradiente de elevación de 300 a 2,500 m snm. En esta área la precipitación oscila entre 800 y 1,600 mm anuales con un promedio de $1,200 \mathrm{~mm}$, mientras que la temperatura entre $16^{\circ} \mathrm{C}$ y $25^{\circ} \mathrm{C}$ (García 1998$)$.

Registros geográficos del ahuehuete. La generación de los modelos de idoneidad de hábitat de la especie se fundamentaron en diferentes fuentes georeferenciadas, entre ellas, el Global Biodiversity Information Facility (GBIF 2019), la Colección de datos del Herbario Nacional de la UNAM (MEXU 2019), la Red Mundial de Información sobre Biodiversidad de la Comisión Nacional para el Conocimiento y uso de la Biodiversidad (CONABIO

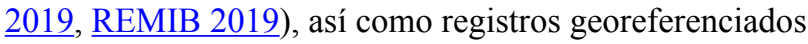
de campo obtenidos mediante estudios dendrocronológicos realizados en el Laboratorio Nacional de Dendrocronología del INIFAP CENID-RASPA.

En total, se obtuvieron 755 registros, los cuales se depuraron mediante la plataforma Niche ToolBox de la CONABIO (Osorio-Olvera et al. 2019). Se eliminaron registros duplicados y con una distancia de separación menor a $1.0 \mathrm{~km}$ lineal entre registros de presencia. El proceso de depuración, evitó el efecto de autocorrelación espacial y por consiguiente una sobreestimación en los modelos de distribución (Peterson \& Nakazawa 2008, Monterrubio-Rico et al. 2016).

Variables climáticas actuales y futuras. La información climática fue obtenida de las 19 capas bioclimáticas existentes de la base de datos WorldClim ver 2.0 (Fick \& Hijmans, 2017), las cuales contienen información climática promedio mundial de 1970 a 2000, con una resolución espacial de 30 segundos $\left(\sim 1 \mathrm{~km}^{2}\right)$ por pixel.

Para el modelado espacial de la idoneidad de hábitat se eligieron los Modelos de Circulación General (MCG) MPIESM-LR y HadGEM2-ES, del Proyecto CMIP-5 (Coupled Model Intercomparison Project Phase 5 2013) (Martin et al. 2011, Giorgetta et al. 2013, IPCC 2013). Se utilizaron dos escenarios de trayectorias de forzamiento radiativo el 4.5 y el 8.5 para los periodos 2041-2060 (2050) y 2061-2080 (2070), bajo una resolución espacial de 30 segundos $\left(\sim 1 \mathrm{~km}^{2}\right)$.
Variables edafológicas y topográficas. La información edafológica se obtuvo de la base de datos SoilGrids (2020), con una resolución espacial de $250 \mathrm{~m}$ (Batjes et al. 2017), información de tipo continuo desarrollada en 2016 por el Global Soil Information Facilities (GSIF). Se extrajeron las variables de fragmentos gruesos (FRA), densidad aparente (DEN), profundidad de suelo (PRO), $\mathrm{pH}(\mathrm{PH})$, capacidad de intercambio catiónico (CIC) y contenido de carbón orgánico (CCO); todas estas variables fueron homogenizadas bajo formato ASCII y tamaño de pixel de 30 segundos $\left(\sim 1 \mathrm{~km}^{2}\right)$.

La información topográfica, se extrajo a partir del modelo digital de elevación (MDE) con una resolución espacial de $120 \mathrm{~m}$ obtenido del continuo de elevaciones mexicano 3.0 (INEGI 2013), del cual se obtuvo la pendiente (PEN) y la orientación (ORI), la información raster se homologó a un tamaño de 30 segundos con formato ASCII.

Selección de variables. La selección de variables se llevó a cabo mediante la generación de un polígono mínimo convexo (PMC), generado a partir de los registros de presencia de la especie (Fitz-Maurice et al. 2013). Se colocaron 10,000 puntos de fondo, con base en el criterio de no discriminación (sin repeticiones) de información relevante para ambientes heterogéneos, dentro del rango de la información climática del ahuehuete y se extrajo la información de cada variable climática, edáfica y topográfica para cada punto de fondo (Becerra-López et al. 2017). Para evitar autocorrelación entre variables, se eliminaron aquellas con correlación mayor a $0.70(P<0.05)$, así evitar el efecto de multicolinearidad entre variables (Merow et al. 2013).

Las variables seleccionadas se delimitaron a una misma resolución espacial de 30 segundos $\left(\sim 1 \mathrm{~km}^{2}\right)$ contemplando el área de modelamiento $\mathrm{M}$ (Figura 1), la cual consiste en el espacio geográfico donde la especie ha sido registrada y está delimitada de acuerdo al conocimiento biológico y a su capacidad de dispersión (Soberón \& Townsend Peterson 2005).

Calibración del modelo. La calibración de los modelos se desarrolló a través del coeficiente estandarizado del criterio de información Akaike (AICc), el cual permite obtener el mejor ajuste de tipo de caracterización, así como el multiplicador de regularización (Warren \& Seifert 2011), mediante la selección de ajuste a través del valor más bajo de ACIc. Dicha calibración se llevó a cabo con la biblioteca ENMeval (Muscarella et al. 2014) del lenguaje y entorno estadístico R v.3.5.3 (R Core Team 2015). 


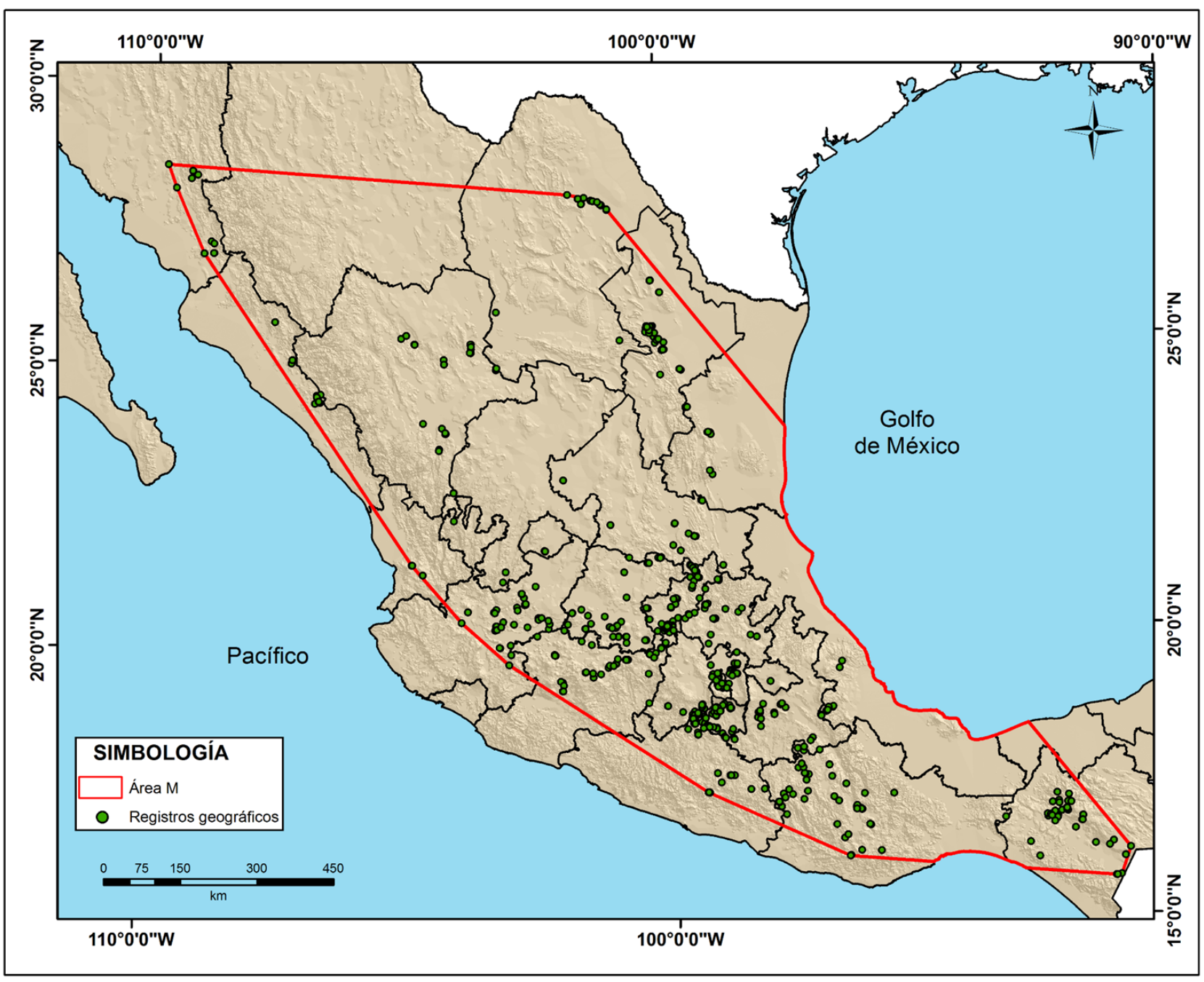

Figura 1. Delimitación de área M y registros de Taxodium mucronatum en México.

Modelación actual. Se utilizó MaxEnt ver. 3.1 .1 (Elith et al. 2006). Las variables seleccionadas después de la depuración fueron BIO11, BIO15, BIO18, BIO19, MDE y PRO en formato ASCII (Tabla 1), los criterios de configuración de MaxEnt consistieron en replicado interno por validación cruzada, (1,000 iteraciones), salida tipo logística, (100 réplicas), un umbral de convergencia de 0.0001 ; en el que se seleccionó $75 \%$ de los registros para entrenar el modelo y $25 \%$ para la validación (Phillips \& Dudik 2008).

El algoritmo de MaxEnt generó un mapa de idoneidad de hábitat con base a sus requerimientos biofísicos, además, se obtuvo un segundo mapa que contempla únicamente las zonas donde se ubican cuerpos de agua o hidrología superficial. Este último se obtuvo con datos vectoriales, mediante un buffer de $15 \mathrm{~m}$ por ambos lados del margen de afluentes perennes, para estimar la super- ficie real con presencia de la especie bajo la premisa de su hábitat ripario y que la banda de vegetación rivereña, difícilmente va más allá de esta distancia.

Modelación bajo escenario a futuro. Para obtener los modelos de idoneidad de hábitat bajo condiciones de cambio climático, los parámetros de calibración y del modelo con mejor ajuste estadístico fueron transferidos al programa MaxEnt ver. 3.4.1 (Morrone \& Escalante 2016).

Para estimar la superficie (ha) de zonas con idoneidad de hábitat actual y futura del ahuehuete, se desarrolló una reclasificación de los valores continuos de ambas proyecciones, mediante tres categorías de idoneidad o probabilidad de hábitat con igualdad de intervalo (baja, media y alta), con el software Arcmap ver. 10.3 a través de la herramienta reclass (Esri 2014). El procedimiento anterior, permitió determinar umbrales de corte y a través de 
la categoría alta, transformar los modelos de continuos a binarios (apto-no apto) para cada periodo evaluado (Manzanilla-Quiñones et al. 2019a). Para obtener un modelo influenciado por condiciones de cambio climático, se promediaron los dos MCG evaluados (MPIESM-LR y HadGEM2-ES), mediante álgebra de mapas en un sistema de información geográfica.

Las áreas que pudieran ser destinadas a conservación de la especie se identificaron a través de un intersecto, entre las áreas de idoneidad de hábitat actual y futura (2070) bajo un escenario RCP 4.5, ya que representa las emisiones actuales de $\mathrm{CO}_{2}$. El geoprocesamiento fue llevado a cabo mediante un análisis espacial de superposición, con la herramienta intersect en Arcmap 10.3. Estas áreas se pueden establecer, debido a la simulación de condiciones ambientales actuales y futuras permisibles para actividades de conservación, reforestación y restauración in situ, considerando los dos criterios de análisis del ahuehuete en México. Además, se estimó la superficie de zonas propuestas para conservación actuales dentro de los polígonos de Áreas Naturales Protegidas (SEMARNATCONANP 2017).

Validación de modelos. Los modelos de distribución, se evaluaron mediante las pruebas estadísticas del área bajo la curva (AUC, por sus siglas en inglés) del análisis de características de operación del receptor (ROC, por sus siglas en inglés); dicha prueba oscila en el rango de $0.0 \mathrm{a}$ 1.0 , donde valores de 0.7 a 0.9 indican un ajuste adecuado $\mathrm{y}$ valores $>0.9$, lo clasifican como excelente (Peterson et al. 2011).

La utilidad del AUC ha sido muy cuestionada para el desarrollo de algoritmos únicamente con datos de presencia, ya que también requiere datos de ausencias verdaderas, por tal motivo, pondera de la misma manera los errores de omisión y comisión (Lobo et al. 2007). Por lo anterior, fue necesario evaluar los modelos mediante el análisis de ROC parcial con la plataforma Niche Tool-
Box de CONABIO (Osorio-Olvera et al. 2019) y de esta manera, contrarrestar las deficiencias del AUC (Peterson \& Nakazawa 2008). En la evaluación de este proceso, se siguieron las recomendaciones de Peterson \& Nakazawa (2008) con 1,000 réplicas por bootstrap con los archivos ASCII para cada periodo y los registros de presencia de la especie, con un error de omisión de $5 \%$.

La prueba de ROC parcial oscila de 1.0 a 2.0, donde un valor medio de radio de 1.0 representa un modelo al azar y un valor de 2.0 representa el mejor desempeño del modelo (Garza-López et al. 2016). Para validar estadísticamente las proporciones de ROC parcial, se efectuaron pruebas de $\mathrm{z}$ de los modelos y se seleccionó el mejor modelo con el valor más alto de las pruebas de ROC parcial, menor error estándar y un valor confiable de Z. Las salidas de los modelos seleccionados para cada periodo se proyectaron a manera de mapas de distribución en el software Arcmap ver. 10.3 (Esri 2014).

Cambio porcentual de superficie por efectos de cambio climático. La evaluación de la tendencia en cambio de superficie ocasionado por efectos del cambio climático, se realizó mediante la aplicación de la ecuación entre periodos sugerida por Gutiérrez \& Trejo (2014). Es decir, la tasa de cambio ocurrida entre el periodo actual y los escenarios a futuro (2050 y 2070), considerando tanto el RCP 4.5 como el 8.5, así como el cambio entre periodos de cambio climático:

$$
\% \text { Cambio }=\left[\frac{\left(S_{1}-S_{0}\right)}{S_{0}}\right] \times 100
$$

donde, $\mathrm{S}_{0}$ es la superficie total, de acuerdo al escenario base, y $\mathrm{S}_{1}$ es la superficie bajo condiciones de cambio climático.

Variables ambientales relevantes. La contribución de las variables ambientales en la distribución actual y futura de la especie, se evaluó a través de la prueba de Jacknife, la

Tabla 1. Variables utilizadas en la generación del modelo de distribución actual del ahuehuete en México.

\begin{tabular}{llc}
\hline Variable & \multicolumn{1}{c}{ Descripción } & Rango (Unidad) \\
\hline BIO11 & Temperatura promedio del trimestre más frío & $-1.68-27.54\left({ }^{\circ} \mathrm{C}\right)$ \\
BIO15 & Estacionalidad de la precipitación (Coeficiente de variación) & $31.60-149.70(\%)$ \\
BIO18 & Precipitación del trimestre más cálido & $25.85-1018.09(\mathrm{~mm})$ \\
BIO19 & Precipitación del trimestre más frío & $2.02-440.54(\mathrm{~mm})$ \\
MDE & Modelo digital de elevación & $0-5556(\mathrm{~m} \mathrm{snm})$ \\
PRO & Profundidad & $0-200(\mathrm{~cm})$ \\
\hline
\end{tabular}



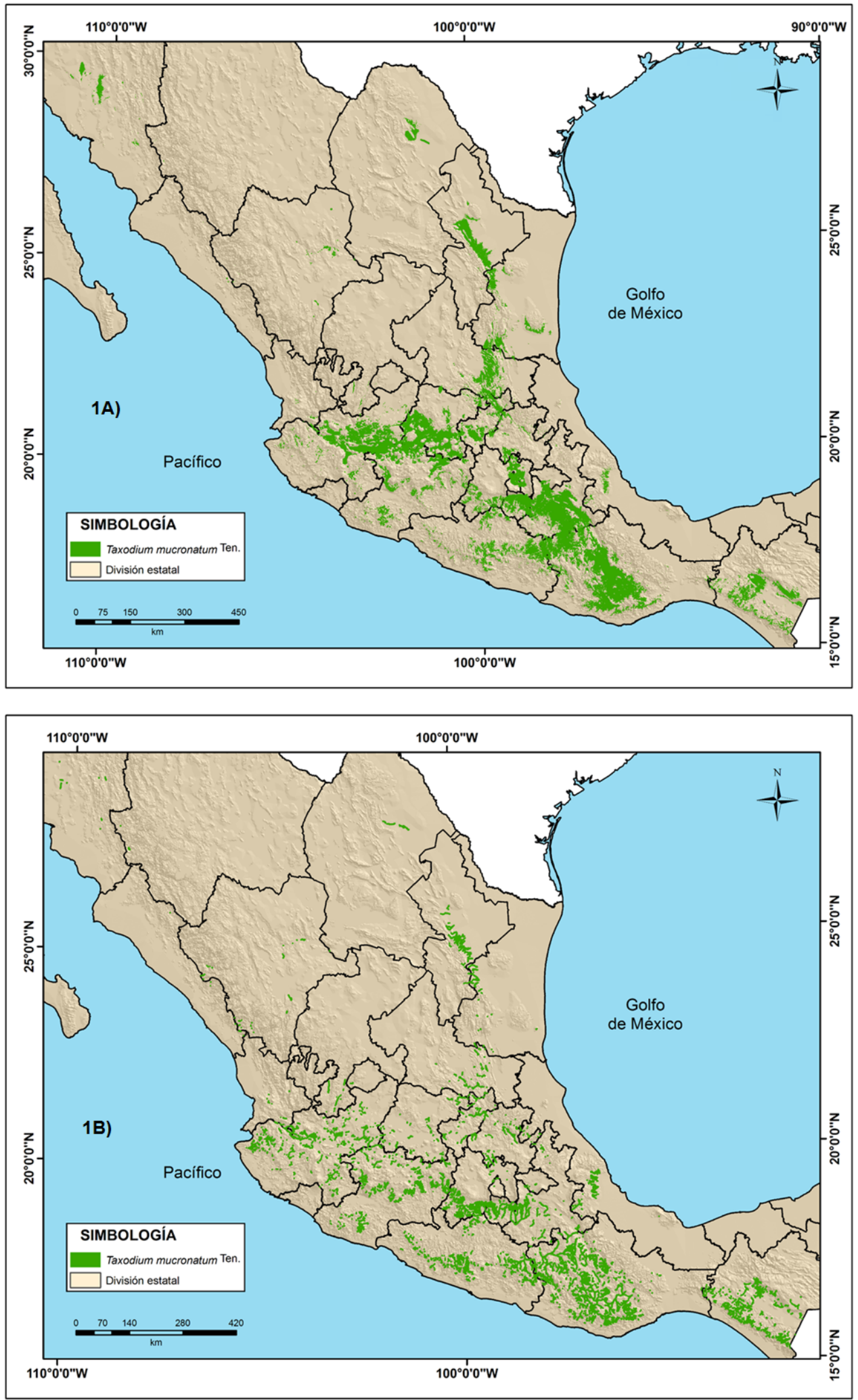

Figura 2. A) Áreas de idoneidad de hábitat actual del ahuehuete en México. B) Áreas al considerar una superficie que se extiende $15 \mathrm{~m}$ a cada lado del margen de corrientes perennes en México. 
Distribución actual y futura de Taxodium mucronatum Ten.

cual permite identificar y cuantificar el porcentaje de contribución de las variables, que favorecen el desarrollo de la especie en México (Phillips et al. 2006).

\section{Resultados}

Modelo de idoneidad de hábitat actual. Las 100 réplicas, de los modelos de idoneidad de hábitat actual, tuvieron valores de AUC entre 0.872 y 0.891 para entrenamiento, y 0.852 a 0.869 para validación, lo que indica que el desempeño de los modelos fue adecuado. El valor de ROC parcial para el mejor modelo fue 1.83, con un AUC de 0.891 para entrenamiento y 0.869 para validación (D.S. $=0.0001$ ).

La superficie actual estimada para el ahuehuete en México fue de 11,217,706.2 ha (Figura 2A) que corresponde a la salida geográfica reclasificada por MaxEnt. Por otro lado, se estimó una superficie de 48,958.5 ha (Figura 2B) al considerar sólo el área bajo un buffer de $15 \mathrm{~m}$ por margen de las corrientes perennes y cuerpos de agua permanentes. Este último valor constituye el área más representativa de la idoneidad de hábitat del ahuehuete en México, con base a su hábitat ripario y la formación de bosques de galería.

La distribución del ahuehuete se concentra principalmente en la región centro-sur de México. Los estados con mayor área de idoneidad de hábitat fueron Oaxaca $(2,430,925.1$ ha, $21.6 \%)$, Jalisco $(1,204,177.5$ ha, $10.7 \%)$ y Puebla $(1,175,218.5$ ha, $10.4 \%)$. Considerando el segundo criterio utilizado, que considera los cuerpos de agua y la vegetación de galería, Oaxaca se mantiene como el estado con mayor superficie $(14,341.8$ ha, $29.2 \%$ ), seguido por Chiapas (4,791.9 ha, 9.7\%), y Michoacán (4,711.2 ha, 9.6 \%). En la Figura S1 (Material suplementario) se muestran las superficies determinadas bajo los dos criterios de clima actual para cada estado.

Modelación bajo cambio climático (RCP 4.5). Los valores obtenidos del AUC de la prueba ROC para el modelo MPIESM-LR, período 2050 fueron de 0.849 a 0.856 para los datos de entrenamiento y de 0.835 a 0.867 para validación, mientras que para el periodo 2070 los valores de AUC fueron de 0.855 a 0.861 para entrenamiento y de 0.845 a 0.872 para validar el modelo. Los resultados del AUC para el modelo HadGEM2-ES para el periodo 2050 fueron de 0.843 a 0.850 para entrenamiento y de 0.825 a 0.860 para validación, mientras que para el periodo evaluado de 2070 fueron de 0.853 a 0.857 para entrenamiento y de 0.842 a
0.869 para validar el modelo. Los valores obtenidos permiten considerar adecuados los modelos de idoneidad de hábitat a futuro.

Modelación bajo cambio climático (RCP 8.5). Los valores obtenidos de AUC del modelo MPIESM-LR del periodo climático 2050 fueron de 0.863 a 0.864 para entrenamiento del modelo y de 0.857 a 0.863 de validación, en el periodo 2070 se obtuvieron valores de AUC de 0.861 a 0.864 y de 0.849 a 0.868 para entrenamiento y validación, respectivamente. Por otra parte el modelo de cambio climático HadGEM2-ES y el periodo 2050, se obtuvieron valores AUC de 0.853 a 0.857 y de 0.833 a 0.855 para entrenamiento $\mathrm{y}$ validación del modelo, respectivamente. Mientras que para la proyección 2070 se obtuvieron valores de 0.855 a 0.858 para entrenamiento y 0.824 a 0.866 para validación. Resultados que permiten clasificar a los modelos de idoneidad de hábitat de ahuehuete como adecuados, bajo un escenario de alta tasa de concentraciones de $\mathrm{CO}_{2}$.

Los valores de ROC para ambos modelos de circulación general y los dos escenarios RCP fluctuaron entre $1.80 \mathrm{y}$ 1.82 , en todos los casos fueron significativos $(P<0.01)$.

Variables relevantes en la distribución actual y futura. Las variables ambientales más relevantes en la distribución actual del ahuehuete en México fueron BIO11, BIO18, MDE, BIO19 y BIO15 (ver Tabla 1), que representan $93.3 \%$ de la variabilidad total del modelo.

Las variables más relevantes considerando el escenario RCP 4.5 para el modelo MPIESM-LR fueron BIO11, MDE, PRO, BIO15 y BIO19 con el $99.0 \%$ y BIO11, MDE, PRO, BIO15 y BIO18 con $99.0 \%$ para 2050 y 2070 , respectivamente. Para el caso del modelo HadGEM2-ES proyectado para 2050 bajo un escenario RCP 4.5, las variables BIO11, MDE, PRO, BIO15 y BIO18 explican $99.0 \%$ de la variación; mientras que las variables BIO11, MDE, PRO, BIO15 y BIO18 explican $98.5 \%$ del modelo para proyección 2070.

Por otra parte, las variables más relevantes bajo un escenario de cambio climático RCP 8.5 y el modelo MPIESM-LR para 2050 y 2070 fueron BIO11, MDE, BIO15, PRO y BIO19 que representan el $98.4 \%$ y $98.0 \%$, respectivamente, de la variabilidad del modelo.

En función al modelo HadGEM2-ES bajo un escenario RCP 8.5, las variables fueron BIO11, MDE, PRO, BIO15 y BIO18 para 2050 y 2070 , que explican el $98.8 \%$ y $98.7 \%$ del total del modelo, respectivamente.

Superficies actual y futura del ahuehuete en México. La superficie estimada actual y futura del ahuehuete en Méxi- 
Martínez-Sifuentes et al. / Botanical Sciences 99(4): 752-770. 2021

Tabla 2. Superficies actual y futura del ahuehuete en México, al considerar los criterios; 1) Idoneidad de hábitat, y 2) Ecosistema ripario.

\begin{tabular}{lcc}
\hline Modelo & Superficie (ha) & Superficie (ha) \\
& Criterio 1 & Criterio 2 \\
\hline Actual & $11,217,706.28$ & $48,958.57$ \\
Cambio Climático (2050; RCP 4.5) & $8,228,125.33$ & $36,543.23$ \\
Cambio Climático (2070; RCP; 4.5) & $4,058,776.32$ & $16,921.47$ \\
Cambio Climático (2050; RCP 8.5) & $3,924,459.11$ & $14,138.53$ \\
Cambio Climático (2070; RCP 8.5) & $3,360,158.67$ & $13,153.63$ \\
\hline
\end{tabular}

co, se presenta en la Tabla 2. La determinación de superficie bajo cambio climático, se determinó al promediar ambos modelos y generar un modelo representativo para los periodos proyectados 2050 y 2070 para para el escenario RCP 4.5 (Figura 3) y el escenario RCP 8.5 (Figura 4). En todas las situaciones, se presenta la superficie bajo los dos criterios establecidos, el de idoneidad de hábitat y el de ecosistema ripario.

Los resultados obtenidos de los modelos de cambio climático considerando un escenario 4.5 para 2050 , reduciría la superficie ocupada de distribución de la especie en $26.6 \%$, con respecto a la superficie actual; de la misma manera, para 2070, se esperaría una disminución de $63.8 \%$ con base a la superficie actual y el primer criterio de distribución. Con base al criterio de ecosistema ripario, la superficie disminuirá $25.3 \%$ en 2050 y $65.4 \%$ en 2070 .

De la misma manera, pero considerando el escenario más pesimista (RCP 8.5), para 2050 se prevé una disminución de $65 \%$ para el primer criterio de análisis y $71.1 \%$ en el segundo criterio. Para 2070 se prevé una disminución de superficie de 70 y $73.1 \%$ para ambos criterios de estudio, de acuerdo a la estimación de superficie de la idoneidad de hábitat de ahuehuete actual para México.

Acorde a la distribución futura del ahuehuete, con respecto a la distribución actual estatal y bajo el primer criterio de estudio con escenario RCP 4.5, las entidades más afectadas para 2050 serían Sonora, Sinaloa y Chihuahua con $-100 \%$, es decir, sin presencia de ahuehuete. También se verían afectados Tlaxcala (-96.7 \%) y Nuevo León (-99.6\%), mientras que se prevé un aumento en superficie para Aguascalientes $(+5,885.5 \%)$ y Colima $(+5,698.9 \%)$. Por otra parte, al considerar la distribución para 2070, los estados más afectados serían Sonora, Coahuila, Sinaloa y Chihuahua (-100\%), Nuevo León $(-99.94 \%)$, Veracruz $(-97.8 \%)$ y Tlaxcala $(-96.79 \%)$, con un aumento en superficie en los estados de Aguascalientes (11,513.53\%), Colima (551.25\%), Zacatecas
(229.11 \%) y Durango (25.02 \%), con respecto a la superficie actual estatal.

Con base al criterio de ecosistema ripario de la distribución estatal actual y los modelos de cambio climático para 2050, se espera que en los estados de Sonora, Tlaxcala, Chihuahua y Sinaloa desaparezca la especie y que haya una disminución para Nuevo León (-99.8 \%) y Tamaulipas (99.4\%); aunque se prevé un aumento en superficie en los estados de Colima (17,171.1\%), Aguascalientes (573.7 \%), Durango (132.9 \%) y Zacatecas (61.6\%). La distribución esperada para 2070, afectaría las entidades de Nuevo León, Tamaulipas, Coahuila, Sonora, Tlaxcala, Chihuahua y Sinaloa (-100 \%), Veracruz (-97.5\%) y Nayarit (-91.0\%) y se predice aumento en superficie del ahuehuete solamente en Aguascalientes (162.9\%) y Colima (75.5\%).

De acuerdo con los resultados del análisis de zonas destinadas a conservación del ahuehuete, para 2070 en base a la integración de los modelos MPIESM-LR y HadGEM2ES con el escenario RCP 4.5, se proponen diversas zonas para actividades de conservación (Figura 5). La superficie propuesta es de 3,207,647.8 ha (basado en el primer criterio), y 14,057.2 ha (basado en el criterio de ecosistema ripario), ambas áreas ubicadas en el centro y sur de México.

Las zonas propuestas para conservación con mayor superficie dentro de las actuales Áreas Naturales Protegidas Federales, Estatales y Municipales se describen en la tabla 3.

Cambio porcentual de superficie por efectos de cambio climático. Considerando el criterio de idoneidad de hábitat para la especie, entre la superficie actual y los efectos esperados del cambio climático para 2050 con el escenario RCP 4.5, se espera un porcentaje de cambio del $-26.6 \%$, y para 2070 de $-63.8 \%$, que implica una diferencia entre escenarios de $-50.6 \%$. De igual manera, acorde al segundo criterio, que considera el hábitat ripario, entre la distribución actual y proyectada para 2050 y 2070, el 
Distribución actual y futura de Taxodium mucronatum Ten.

porcentaje de cambio sería de -25.3 y $-65.4 \%$, respectivamente, y entre modelos a futuro sería de $-53.6 \%$. Para 2050 ante un escenario de cambio climático RCP 8.5 , se espera una reducción de $65 \%$ y para 2070 de $70 \%$ bajo el primer criterio, lo que implica una disminución de $14.3 \%$ entre periodos. Analizando el segundo criterio, se prevé para 2050 disminución de $71.1 \%$ y para 2070 $73.13 \%$, lo que implica una tasa de $6.96 \%$ entre ambos periodos.

\section{Discusión}

Modelado espacial. El presente estudio constó de 633 registros de presencia de ahuehuete posterior al proceso de depuración. El aumento en la precisión para el modelado de áreas de idoneidad de hábitat de especies, se obtiene al considerar una cantidad mayor a 50 registros (Stockwell
\& Townsend Peterson 2002), aunque un modelado de especies por encima de 470 registros es idóneo para el proceso de entrenamiento y la prueba de validación (AcevesRangel et al. 2018).

El estudio de ahuehuete bajo los dos criterios analizados, constató su presencia en las entidades federativas de la República Mexicana donde se ha registrado y documentado su distribución. Es así que se corrobora resultados de estudios pioneros que indican que la especie tiene una amplia distribución en México, excepto en las penínsulas de Baja California y Yucatán (Martínez 1963). En México, se ha desarrollado una serie de estudios para modelar la presencia de especies en condiciones climáticas actuales (Martínez-Méndez et al. 2016, Aceves-Rangel et al. 2018, Manzanilla-Quiñones et al. 2019b) y para escenarios de cambio climático (Pérez-Miranda et al. 2019, Araiza-Olivares 2020, Manzanilla-Quijada et al. 2020b); sin embar- a)
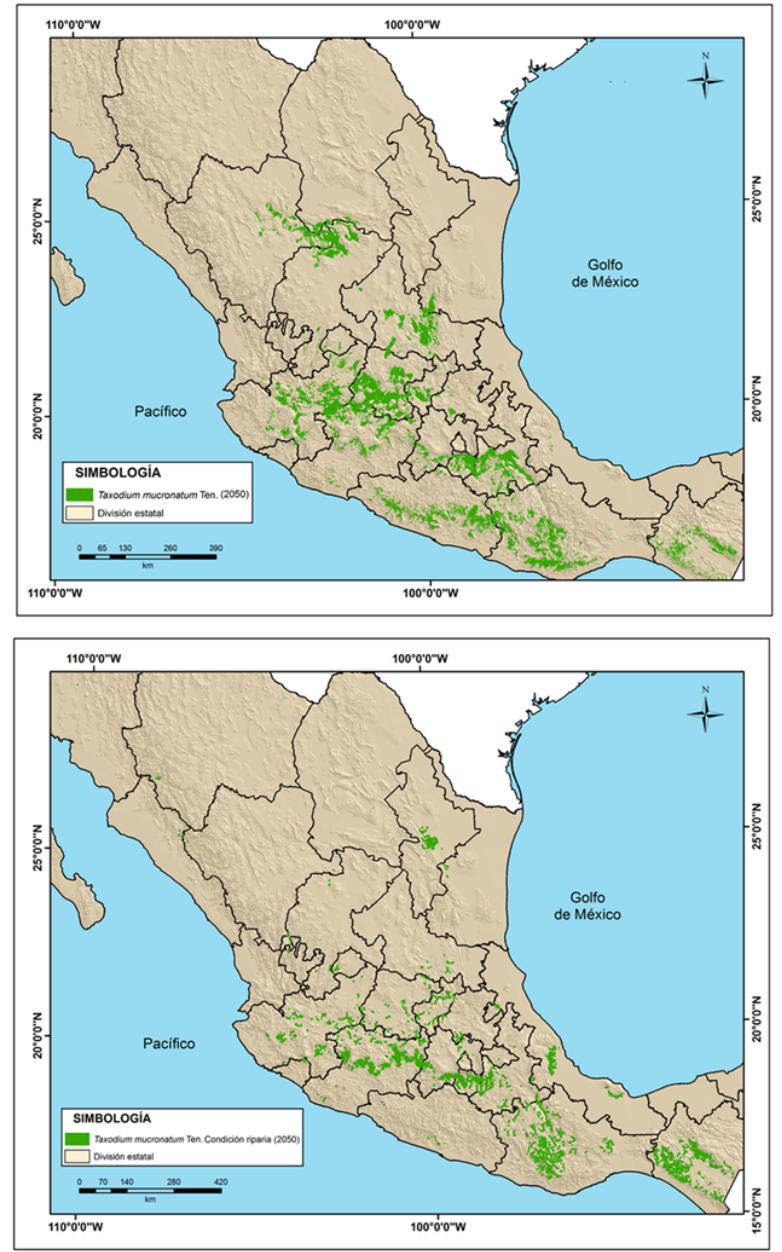

b)
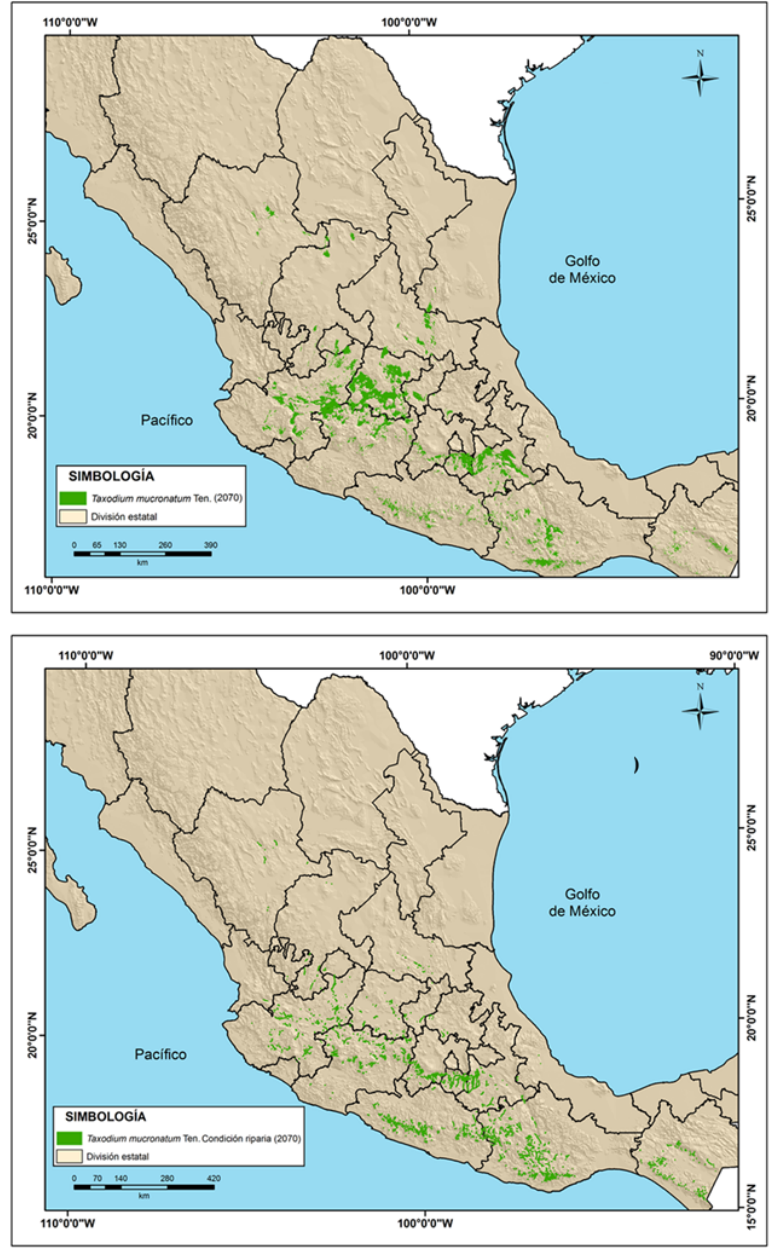

Figura 3. Mapas de idoneidad de hábitat de ahuehuete en México para A) 2050 y B) 2070, bajo el escenario RCP 4.5, considerando los criterios de idoneidad de hábitat (imagen superior), y condición riparia (imagen inferior). 
Tabla 3. Superficie estimada de zonas propuestas para conservación a través del análisis de superposición, dentro de Áreas Naturales Protegidas decretadas en la actualidad.

\begin{tabular}{|c|c|c|c|}
\hline \multicolumn{2}{|l|}{ Criterio de distribución potencial } & \multicolumn{2}{|c|}{ Criterio de ecosistema ripario } \\
\hline Área Natural Protegida & $\begin{array}{l}\text { Superficie } \\
\text { (ha) }\end{array}$ & Área Natural Protegida & Superficie (ha) \\
\hline $\begin{array}{l}\text { Cuencas de los Ríos Valle de Bravo, Mala- } \\
\text { catepec, Tilostco y Temascaltepec (Feder- } \\
\text { al; Estado de México y Michoacán) }\end{array}$ & $21,761.96$ & $\begin{array}{l}\text { Cuencas de los Ríos Valle de Bravo, } \\
\text { Malacatepec, Tilostco y Temascaltepec } \\
\text { (Federal; Estado de México y Micho- } \\
\text { acán) }\end{array}$ & 392.73 \\
\hline La Sepultura (Federal; Chiapas) & $8,916.97$ & $\begin{array}{l}\text { Parque Ecológico Recreativo de Ten- } \\
\text { ancingo, Malinalco y Zumpahuacán (Es- } \\
\text { tatal; Estado de México) }\end{array}$ & 110.35 \\
\hline $\begin{array}{l}\text { Presa de Silva y sus Áreas Aledañas (Es- } \\
\text { tatal; Guanajuato) }\end{array}$ & $8,677.11$ & La Sepultura (Federal; Chiapas) & 102.62 \\
\hline $\begin{array}{l}\text { Terrenos que se encuentran en los mu- } \\
\text { nicipios de La Concordia, Ángel Albino } \\
\text { Corzo, Villa Flores y Jiquipilas (Federal; } \\
\text { Chiapas) }\end{array}$ & $8,458.65$ & $\begin{array}{l}\text { Santuario del Agua Valle de Bravo (Es- } \\
\text { tatal; Estado de México) }\end{array}$ & 93.18 \\
\hline $\begin{array}{l}\text { Corredor Biológico Chichinautzin (Fed- } \\
\text { eral; Ciudad de México, Morelos y Estado } \\
\text { de México) }\end{array}$ & $8,334.51$ & $\begin{array}{l}\text { Terrenos que se encuentran en los mu- } \\
\text { nicipios de La Concordia, Ángel Albino } \\
\text { Corzo, Villa Flores y Jiquipilas (Federal; } \\
\text { Chiapas) }\end{array}$ & 92.56 \\
\hline $\begin{array}{l}\text { El Tepozteco (Federal; Morelos y Ciudad } \\
\text { de México) }\end{array}$ & $7,395.81$ & $\begin{array}{l}\text { Cordón Pico El Loro-Paxtal (Estatal; } \\
\text { Chiapas) }\end{array}$ & 66.07 \\
\hline Reserva de la Biósfera Tehuacán-Cuicatlán & $7,274.80$ & $\begin{array}{l}\text { Reserva de la Biósfera El Triunfo (Fed- } \\
\text { eral; Chiapas) }\end{array}$ & 27.79 \\
\hline $\begin{array}{l}\text { Parque Ecológico Recreativo de Tenancin- } \\
\text { go, Malinalco y Zumpahuacán (Estatal; Es- } \\
\text { tado de México) }\end{array}$ & $5,413.41$ & $\begin{array}{l}\text { Zona Sujeta a Conservación Ecológica } \\
\text { Los Sabinos-San Cristóbal (Estatal; Mo- } \\
\text { relos) }\end{array}$ & 24.76 \\
\hline Humedal del Valsequillo (Estatal; Puebla) & $5,260.03$ & $\begin{array}{l}\text { El Tepozteco (Federal; Morelos y Ciudad } \\
\text { de México) }\end{array}$ & 16.46 \\
\hline $\begin{array}{l}\text { Reserva de la Biósfera El Triunfo (Federal; } \\
\text { Chiapas) }\end{array}$ & $5,185.78$ & $\begin{array}{l}\text { Parque Natural Nahuatlaca-Matlazinca } \\
\text { (Estatal; Estado de México) }\end{array}$ & 15.83 \\
\hline
\end{tabular}


A)


B)
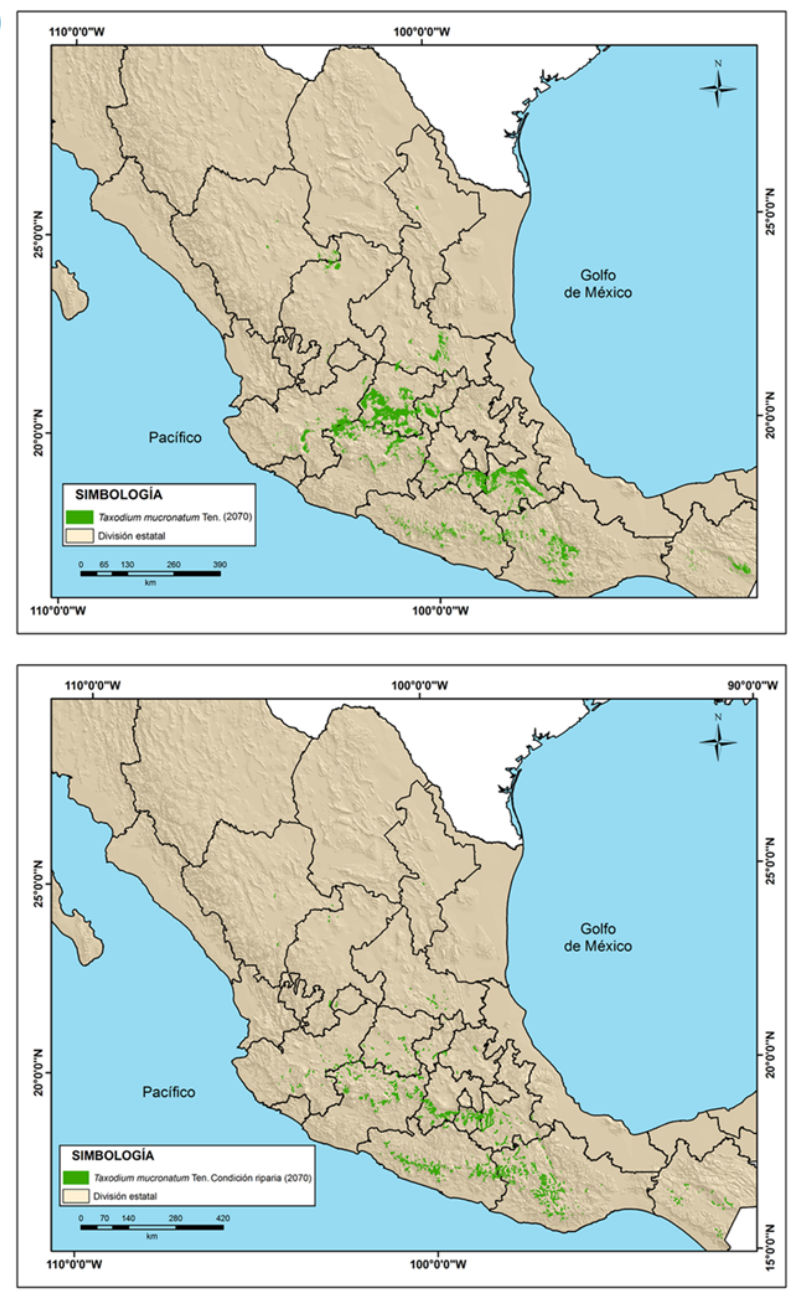

Figura 4. Mapas de idoneidad de hábitat de ahuehuete en México para A) 2050 y B) 2070, bajo el escenario RCP 8.5, considerando los criterios de idoneidad de hábitat (imagen superior), y condición riparia (imagen inferior).

go, no se habían realizado estudios sobre la distribución potencial del ahuehuete, a pesar de su importancia cultural y ecológica. Diversos estudios se han realizado sobre esta especie en otros como análisis estructurales (Suzán-Aspiri et al. 2007, Alanís-Rodríguez et al. 2020), concentración de isótopos de carbono (Beramendi-Orosco et al. 2018), presencia de metales pesados (Morton-Bermea et al. 2016), reconstrucción climática en norte-centro de México (Villanueva-Díaz et al. 2020) y como bioindicador de $\mathrm{CO}_{2}$ en la atmósfera (Correa-Díaz et al. 2018).

La superficie de bosques de galería en México es de aproximadamente 2,600,000 ha (Rzedowski 1986). Sin embargo, con base a datos de INEGI (2016), esta superficie inicial se ha reducido a cerca de 177,244.6 ha, incluye toda especie de vegetación riparia de México con o sin ahuehuete. En este estudio el área estimada de bosque de galería con presencia del ahuehuete es de 48,958.5 ha. La disminución de los ecosistemas riparios se atribuye en alta medida a cambios en el uso del suelo, construcción de presas, derivación de volúmenes de agua con fines agrícolas y problemas de contaminación de las corrientes principales donde se distribuye la especie (Arriaga et al. 2000, Ceccon 2003, Villanueva-Díaz et al. 2014).

Variables ambientales relevantes. La variable BIO11 (temperatura promedio del trimestre más frío) fue la más relevante para la presencia actual del ahuehuete en México, información que coincide con diversos estudios relacionados al hábitat de coníferas en México, donde esta variable ha demostrado su importancia en el hábitat de especies como Pinus engelmani, Pinus arizonica, Pinus nelsonii y Abies religiosa (Martínez-Méndez et al. 2016, 
Aceves-Rangel et al. 2018, García-Aranda et al. 2018). No obstante, que el rango óptimo para el desarrollo del ahuehuete en México oscila entre 16 a $22{ }^{\circ} \mathrm{C}$ en climas templados y semicálidos (CONABIO 2019). La especie es indiferente a la temperatura, siempre y cuando exista una fuente constante de humedad, como pueden ser nacimientos de agua, sitios con agua muy superficial o corrientes perennes o semi-permanentes.

Cuando existe una fuente de humedad continua, el factor que limita el crecimiento de la especie es la temperatura y esto se confirma por la limitada actividad fisiológica del ahuehuete en el período invernal, cuando entra en dormancia sus procesos se activan con el incremento en la temperatura que ocurren al final de la etapa invernal y principios de primavera para el norte y centro de México (Villanueva-Díaz et al. 2013). El efecto fisiológico de estas bajas temperaturas, parece ser la inducción en la formación de ciertas hormonas del crecimiento, como lo es el ácido abscísico, que contribuye a que la especie tolere el estrés hídrico, al reducir la pérdida de agua por transpiración ( $\mathrm{Wu}$ et al. 1997).

La variabilidad en la temperatura es un común denominador en el modelado espacial de especies de plantas vasculares terrestres (Aceves-Rangel et al. 2018, ManzanillaQuiñones et al. 2019a). Este comportamiento, se atribuye a que temperaturas elevadas, promueven un incremento de evapotranspiración y por consiguiente, una alteración metabólica que impacta la asimilación de fotosintatos (Girardin et al. 2012). La temperatura es un componente importante en el cambio climático global y ha presentado cambios en su variabilidad (Medhaug et al. 2017), especialmente en el norte de México, donde los modelos climáticos pronostican incrementos significativos en esta variable (Abram et al. 2019). Lo anterior hace suponer que el crecimiento podría ocurrir más temprano de lo habitual, pero con reducción en incremento radial y producción de biomasa (Gennaretti et al. 2014).

La variable BIO18 (precipitación del trimestre más cálido), es una variable importante para el establecimiento y crecimiento de especies arbóreas, como lo ha demostrado diversos estudios con especies del género Abies en México (Martínez-Méndez et al. 2016). En este estudio, esta variable fue de importancia en ahuehuete; situación que pudiera tener un efecto indirecto, ya que mayor precipitación en el verano se relaciona con mayores volúmenes de caudales en los tributarios y corrientes principales, que favorecen la oxigenación del agua, promoviendo así un mayor crecimiento de la especie al ser de hábitat ripario (Suzán-Aspiri et al. 2007). Otra característica de la es- pecie es de que, a pesar de vivir aledaño a las corrientes de agua, gran parte de sus raíces se encuentran fuera del cauce, por lo que depende del agua almacenada en el perfil del suelo, humedad que es más fácilmente aprovechada en el período invierno-primavera y verano, lo que favorece un mayor incremento radial (Villanueva Díaz et al. 2003, Villanueva-Díaz et al. 2020). En este estudio, los rangos para la variable BIO18 fueron de 25.8 a $1,018.1 \mathrm{~mm}$, aunque los valores óptimos reportados para la especie se encuentran en el rango de 800 a 1,600 mm (CONABIO 2019).

La variable MDE (modelo digital de elevación), indicó una idoneidad de hábitat de la especie en un rango de 350 a 2,900 $\mathrm{m}$ snm con un valor de $17.1 \%$, aunque el rango adecuado para su desarrollo es de 300 a 2,800 m (Martínez 1963). La elevación es una variable importante para la presencia de diversas especies de coníferas, donde puede explicar cerca del $30 \%$ para el caso de Pinus lawsonii (Aceves-Rangel et al. 2018) y $29.0 \%$ para Pinus montezumae (Manzanilla-Quiñones et al. 2019b).

La variable BIO19 (precipitación del trimestre más frío) explicó $14.6 \%$ en el modelo de hábitat del ahuehuete. Aunque la precipitación en este período puede ser inferior al $10 \%$ del total anual (García 1998), las bajas temperaturas dan pie a una baja evaporación. La lluvia ocurrida en este período queda almacena en el perfil del suelo y posteriormente aprovechada por la especie al inicio de la estación de crecimiento, así promoviendo su presencia en sitios donde la lluvia invernal es significativa (VillanuevaDíaz et al. 2013).

La estacionalidad de la precipitación (BIO15), mostró un valor de importancia en el modelo actual del ahuehuete de $6.9 \%$. La estacionalidad de la precipitación tanto en el período invernal y en el verano, tiene importancia significativa en la distribución del ahuehuete en México. Las lluvias de verano favorecen mayores caudales en las corrientes fluviales donde se ubica la especie; esta condición, favorece una mayor oxigenación del agua en comparación a períodos con bajo caudal o donde el agua se encuentra estancada o con movimiento mínimo por períodos considerables, situación que reduce el crecimiento por falta de oxigenación (Tiner 1999), lo que conduce a la presencia de individuos longevos (Villanueva-Díaz et al. 2010). Lluvias invernales promueven un rápido desarrollo de la especie al inicio de la estación de crecimiento (CorreaDíaz et al. 2014).

Escenarios a futuro y áreas de conservación de nicho. Los MCG elegidos, han sido utilizados en análisis de ido- 
Distribución actual y futura de Taxodium mucronatum Ten.
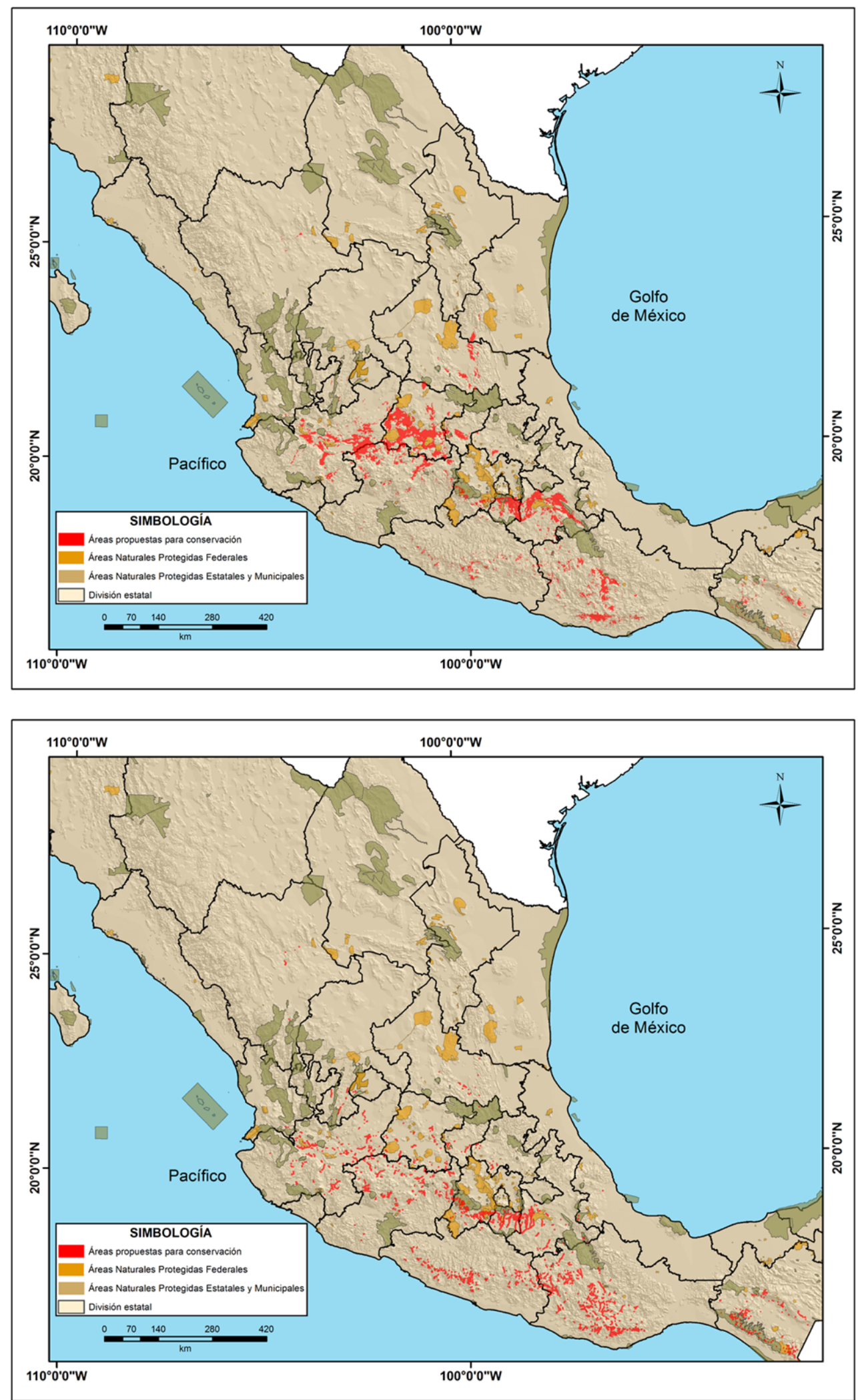

Figura 5. Zonas propuestas para conservación del ahuehuete en México, bajo la proyección a futuro de 2070, considerando el criterio de idoneidad de hábitat (imagen superior) y ecosistema ripario (imagen inferior). 
neidad de hábitat de especies arbóreas, ya que presentan las condiciones climáticas características de la zona de geográfica en el presente estudio (Pinedo-Alvarez et al. 2019). Los resultados obtenidos por los MCG, muestran tendencias similares a los de Martínez-Sifuentes et al. (2020), donde encontraron reducciones de nicho ecológico para Pinus greggii Engelm de $-9.5 \%$, con el modelo HadGEM2-ES bajo dos escenarios de cambio climático. Con respecto al modelo MPIESM-LR, el área potencial ante el cambio climático resultó menor, en comparación al modelo HadGEM2-ES, comportamiento que se ha evidenciado en otros estudios (Vargas-Piedra et al. 2020).

Se ha determinado, que un incremento en temperatura causado por efectos de cambio climático podría generar un impacto negativo en las superficies ocupadas por los géneros Abies, Quercus y Pinus en México (Gutiérrez \& Trejo 2014, Martínez-Méndez et al. 2016, ManzanillaQuiñones et al. 2019a). Esta situación también se comparte para las poblaciones de ahuehuete, de las cuales se estima una reducción de $26.6 \%$ y del $63.8 \%$ para los años 2050 y 2070 , respectivamente.

Los escenarios de cambio climático para 2050 y 2070, indican una alta reducción de las poblaciones actuales de ahuehuete, lo cual sugiere la implementación de acciones con miras a la conservación de esta especie de importancia cultural e histórica en el país. No obstante que, los escenarios de cambio climático predicen reducción de superficie en el nicho ecológico de la especie, también señalan que en la región de convergencia de los estados de Durango, Coahuila y Zacatecas en el norte de México, las condiciones son adecuadas para la especie para 2050. Mientras que las mejores condiciones ocurrirán en el centro-sur de Guanajuato para 2070.

La combinación de altas temperaturas y menor disponibilidad constituirían las causas principales de la reducción del hábitat del ahuehuete en su rango de distribución natural en México. No obstante que no existe un estudio base de distribución del ahuehuete en el país, este patrón de reducción indica una similitud con otras especies forestales, como Cedrela odorata con alto requerimiento hídrico y con amplia distribución en las zonas tropicales de México (Hernández-Ramos et al. 2018, ManzanillaQuijada et al. 2020b).

En la actualidad, el uso de sistemas de información geográfica aunado a modeladores de nicho ecológico como MaxEnt, ha permitido generar cartografía para establecer áreas de conservación natural de especies bajo alguna categoría de riesgo, ya sea que se encuentren en el listado de especies de la Norma Oficial Mexicana
NOM-059, o que en los últimos años sus poblaciones se encuentren en decremento (Garza-López et al. 2016). En este sentido, diversos han sido los estudios de análisis geoespacial para identificar áreas de conservación de especies forestales, tanto en México (Manzanilla-Quijada et al. 2020a), como en el extranjero (Hu et al. 2020). Por tal motivo, es importante apostar por este tipo de estudios que puedan aportar herramientas para la toma de decisiones, ante escenarios climáticos adversos (Jayasinghe \& Kumar 2019).

Algunos estudios indican que de $70 \%$ de las 56 especies modeladas en México, solo $10 \%$ de su distribución natural se resguarda dentro del área poligonal dentro de alguna área natural protegida (Aguirre-Gutiérrez \& Duivenvoorden 2010). Para este estudio ocurre algo similar, ya que solamente $7.6 \%$ del área de distribución natural del ahuehuete, al considerar los criterios de idoneidad de hábitat, se encuentra dentro de algún área natural protegida en México. Pero si se considera el criterio de ecosistema ripario el porcentaje se reduce a solo $0.1 \%$. Fuera de estas áreas, se ubican poblaciones de ahuehuete en condiciones ecológicas de hábitat idóneo, donde se puede promover su conservación, como es el caso de poblaciones en Puebla, Estado de México y Chiapas, que son entidades donde se ubica la mayor superficie idónea para la conservación de esta especie.

Los hallazgos del presente estudio resultan de gran relevancia científica, ya que permiten delimitar y proponer diversas zonas prioritarias para su conservación y restauración del hábitat del ahuehuete, ya sea dentro o fuera de áreas naturales protegidas.

\section{Agradecimientos}

El presente estudio fue apoyado con recursos del Consejo Nacional de Ciencia y Tecnología, mediante el proyecto APN-2976, Apoyado por el FOINS. Fondo Problemas Nacionales "Bosques de galería con ahuehuete (Taxodium mucronatum Ten.): tasas de crecimiento y análisis de la variabilidad hidroclimática y antropogénica con fines de conservación" y Fondo Sectorial de Investigación para la Educación, proyecto CB-2016-01-283134.

\section{Material suplementario}

El material suplementario de este artículo puede ser consultado en la siguiente liga: https://doi.org/10.17129/ botsci. 2772 
Distribución actual y futura de Taxodium mucronatum Ten.

\section{Literatura citada}

Abram N, Gattuso JP, Prakash A, Cheng L, Chidichimo MP, Crate S, Enomoto H, Garschagen M, Gruber N, Harper S, Holland E, Kudela RM, Rice J, Steffen K, Schuckmann K. 2019. Framing and Context of the Report. In: Pörter HO, Roberts DC, Masson-Delmonte V, Zhai P, Tignor M, Poloczanska E, Mintenbeck K, Alegría A, Nicolai M, Okem A, Petzold J, Rama B, Weyer NM, eds. The Ocean and Cryosphere in a Changing Climate. A Special Report of the Intergovernmental Panel on Climate Change, pp. 73-131 https://www.ipcc.ch/ site/assets/uploads/sites/3/2019/12/SROCC_FullReport_FINAL.pdf (accessed January 08, 2021)

Aceves-Rangel LD, Méndez-González J, García-Aranda MA, Nájera-Luna JA. 2018. Potential distribution of 20 pine species in Mexico. Agrociencia 52: 1043-1057.

Aguirre-Gutiérrez J, Duivenvoorden JF. 2010. Can we expect to protect threatened species in protected areas? A case study of the genus Pinus in Mexico. Revista Mexi-

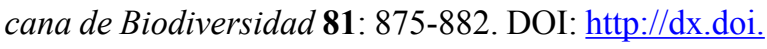
org/10.22201/ib.20078706e.2010.003.657

Alanís-Rodríguez E, Rubio-Camacho EA, CanizalesVelázquez PA, Moro-Olivo A, Pequeño-Ledezma MA, Buendía-Rodríguez E. 2020. Estructura y diversidad de un bosque de galería en el noreste de México. Revista Mexicana de Ciencias Forestales 11: 135-153. DOI: https://doi.org/10.29298/rmcf.v11i58.591

Araiza-Olivares GA. 2020. Efectos del cambio climático en la distribución de oyamel. Revista Geográfica de América Central 65: 263-282. DOI: https://doi. org/10.15359/rgac.65-2.11

Arriaga L, Espinoza JM, Aguilar C, Martínez E, Gómez L, Loa E. 2000. Regiones terrestres prioritarias de México. México: Comisión Nacional Para el Conocimiento y Uso de la Biodiversidad. http://www.conabio.gob. $\mathrm{mx} /$ conocimiento/regionalizacion/doctos/terrestres. $\underline{\mathrm{html}}$ (accessed January 08, 2021)

Batjes NH, Ribeiro E, Oostrum AV, Leenaars J, Hengl T, Mendes de Jesus J. 2017. WoSIS: Providing standardised soil profile data for the world. Earth System Science Data 9: 1-14. DOI: https://doi.org/10.5194/ essd-9-1-2017

Becerra-López JL, Esparza-Estrada CE, Romero-Méndez U, Sigala-Rodríguez JJ, Mayer-Goyenechea IG, Castillo-Cerón JM. 2017. Evidence of niche shift and invasion potential of Lithobates catesbeianus in the habitat of Mexican endemic frogs. Plos One 12: e0185086. DOI: https://doi.org/10.1371/journal.pone. 0185086
Beramendi-Orosco LE, González-Hernández G, Martínez-Reyes A, Morton-Bermea O, Santos-Arévalo FJ, Gómez-Martínez I, Villanueva-Díaz J. 2018. Changes in $\mathrm{CO} 2$ emission sources in Mexico City metropolitan area deduced from radiocarbon concentrations in tree rings. Radiocarbon 60: 21-34. DOI: https://doi. org/10.1017/RDC.2017.100

Carranza E. 1992. Taxodiaceae. In: Rezedowski J, Calderón de Rezedowski G. Flora del Bajio y de Regiones Adyacentes. Pátzcuaro, Michoacán, México: Instituto de Ecología, A.C. Centro Regional del Bajío. Fascículo 4. pp. 1-7. ISBN: 970-709-038-3

Ceccon E. 2003. Los bosques ribereños y la restauración y conservación de las cuencas hidrográficas. Ciencia 72: 46-53.

Correa-Díaz A, Gómez-Guerrero A, Villanueva-Díaz J, Castruita-Esparza LU, Martínez-Trinidad T, Cervantes-Martínez R. 2014. Análisis dendroclimático de ahuehuete (Taxodium mucronatum Ten.) en el centro de México. Agrociencia 48: 537-551.

Correa-Díaz A, Gómez-Guerrero A, Villanueva-Díaz J, Silva L, Horwath W, Castruita-Esparza LU, MartínezTrinidad T, Suárez-Espinosa J. 2018. Respuesta fisiológica de Taxodium mucronatum Ten. a los incrementos atmosféricos de $\mathrm{CO}_{2} \mathrm{y}$ temperatura del último siglo. Agrociencia 52: 129-149.

CONABIO [Comisión Nacional para el Conocimiento y Uso de la Biodiversidad]. 2019. Paquetes tecnológicos. Taxodium mucronatum Ten. Ciudad de México, México. http://www.conafor.gob.mx:8080/documentos/docs/13/1011Taxodium\%20mucronatum.pdf (accessed May 8, 2020)

CONAFOR [Comisión Nacional Forestal]. 2009. Restauración de ecosistemas forestales: guía básica para comunicadores. Zapopan, Jalisco, México: Comisión Nacional Forestal-SEMARNAT. pp. 63. http://www.conafor.gob.mx:8080/documentos/ docs/7/579Restauraci\%C3\%B3n\%20de $\% 20$ ecosistemas\%20forestales.pdf (accessed May 14, 2020)

Cruz-Cárdenas G, López-Mata L, Silva JT, Bernal-Santana N, Estrada-Godoy F, López-Sandoval J A. 2016. Potential distribution model of Pinaceae species under climate change scenarios in Michoacán. Revista Chapingo Serie Ciencias Forestales y del Ambiente 22: 135-148. DOI: https://doi.org/10.5154/r.rchscfa.2015.06.027

Davis MB, Shaw RG. 2001. Range shifts and adaptative response to Quaternary climate change. Science 292: 673-679. DOI: https://doi.org/10.1126/science. 292.5517 .673 
Denny GC, Arnold MA, 2007. Taxonomy and nomenclature of Baldcypress, Pondcypress, and Montezuma Cypress: One, two, or three species? HortTechnology 17: 125-127. DOI: https://doi.org/10.21273/HORTTECH.17.1.125

Elith J, Graham CH, Anderson RP, Dudík M, Ferrier-A, Guisan A, Hijmans RJ, Huettmann F, Leathwick JR, Lehmann A, Li J, Lohmann LG, Loiselle BA, Manion G, Moritz C, Nakamura M, Nakazawa Y, Overton JM, Peterson AT, Phillips SJ, Richardson K, Scachetti-Pereira R, Schapire RE, Soberón J, Williams S, Wisz MS, Zimmermann NE. 2006. Novel methods improve prediction of species distributions from occurrence data. Ecography 29: 129-151. DOI: https://doi. org/10.1111/j.2006.0906-7590.04596.x

Enríquez-Peña EG, Suzán-Azpiri H. 2011. Estructura poblacional de Taxodium mucronatum en condiciones contrastantes de perturbación en el estado de Querétaro, México. Revista Mexicana de Biodiversidad 82: 153-167. DOI: https://doi.org/10.22201/ ib.20078706e.2011.1.380

Esri 2014. ArcGIS Desktop: Release 10.4. Redlands, CA: Environmental System Research Institute

Fick SE, Hijmans RJ. 2017. Worldclim 2: New 1-km spatial resolution climate surfaces for global land areas. International Journal of Climatology 37: 4302-4315. DOI: https://doi.org/10.1002/joc.5086

Fitz-Maurice B, Sotomayor M, Fitz-Maurice WA, Hernández H, Smith M. 2013. Astrophytum coahuilense (Bonete de Obispo); Distribución Conocida; Catálogo de Metadatos Geográficos/Comisión Nacional para el Conocimiento y Uso de la Biodiversidad (CONABIO) México: 2013. http://www.conabio.gob.mx/informacion/gis (accessed April 23, 2020)

García E. 1998. Isotermas Medias Anuales. Escala 1:1000000, Aguascalientes, Aguascalientes, México. http://www.conabio.gob.mx/informacion/gis/ (accessed May 11, 2020)

García-Aranda MA, Méndez-González J, Hernández-Arizmendi JY. 2018. Distribución potencial de Pinus cembroides, Pinus nelsonii y Pinus culminicola en el Noreste de México. Ecosistemas y Recursos Agropecuarios 5: 3-13. DOI: https://doi.org/10.19136/era.a5n13.1396

Garza-López M, Ortega-Rodríguez JM, Zamudio-Sánchez FJ, López-Toledo JF, Domíguez-Álvarez FA, SáenzRomero C. 2016. Calakmul como refugio de Swietenia macrophylla King ante el cambio climático. Botanical Sciences 94: 43-50. DOI: http://dx.doi.org/10.17129/ botsci.500
GBIF [Global Biodiversity Information Facility]. 2019. Global Biodiversity Information Facility. Denmark: Finnish Biodiversity Information Facility. DOI: https:// doi.org/10.15468/dl.fpwlzt

Gennaretti F, Arseneault D, Nicault A, Perreault L, Begin Y. 2014. Volcano-induced regime shifts in millennial tree-ring chronologies from northeastern North America. Proceedings of the National Academy of Sciences of the United States of America, 111: 10077-10082. DOI: https://doi.org/10.1073/pnas.1324220111

Giorgetta MA, Jungclaus J, Reick CH, Legutke S, Bader J, Böttinger M, Brovkin V, Crueger T, Esch M, Fieg K, Glushak K, Gayler V, Haak H, Hollweg HD, Ilyina T, Kinne S, Kornblueh L, Matei D, Mauritsen T, Mikolajewicz U, Mueller W, Notz D, Pithan F, Raddatz T, Rast S, Redler R, Roeckner E, Schmidt H, Schnur R, Segschneider J, Six KD, Stockhause $\mathrm{M}$, Timmreck $\mathrm{C}$, Wegner J, Widmann $\mathrm{H}$, Wieners KH, Claussen M, Marotzke J, Stevens N. 2013. Climate and carbon cycle changes demo1850 to 2100 in MPI-ESM simulations for the Coupled Model Intercomparison Project phase 5. Journal of Advances in Modeling Earth Systems, 5: 572-597. DOI: https://doi. org/10.1002/jame.20038

Girardin MP, Guo XJ, Bernier PY, Raulier F, Gauthier S. 2012. Changes in growth of pristine boreal North American forests from 1950 to 2005 driven by landscape demographics and species traits. Biogeosciences 9: 2523-2536. DOI: https://doi.org/10.5194/bg-92523-2012

Guisan A, Zimmermann NE. 2000. Predictive habitat distribution models in ecology. Ecological Modelling 135: 147-186. DOI: https://doi.org/10.1016/S0304$\underline{3800(00) 00354-9}$

Gutiérrez E, Trejo I. 2014. Efecto del cambio climático en la distribución potencial de cinco especies arbóreas de bosque templado en México. Revista Mexicana de Biodiversidad 85: 179-188. DOI: https://doi.org/10.7550/ $\underline{\mathrm{rmb} .37737}$

Hernández-Ramos J, Reynoso-Santos R, HernándezRamos A, García-Cuevas X, Hernández-Máximo E, Cob-Uicab JV, Sumano-López D. 2018. Distribución histórica, actual y futura de Cedrela odorata en México. Acta Botanica Mexicana 124.

Hernández-Ruíz J, Herrera-Cabrera BE, Delgado-Alvarado A, Salazar-Rojas VM, Bustamante-González A, Campos-Contreras JE, Ramírez-Juárez J. 2016. Distribución potencial y características geográficas de poblaciones silvestres de Vanilla planifolia (Orchidaceae) en 
Distribución actual y futura de Taxodium mucronatum Ten.

Oaxaca, México. Revista de Biología Tropical 64: 235246. DOI: https://doi.org/10.15517/rbt.v64i1.17854

Hession WC, Johnson TE, Charles DF, Hart DD, Horwitz RJ, Kreeger DA, Pizzuto JE, Velinsky DJ, Newbold JD, Cianfrani C, Clason T, Compton AM, Coulter N, Fuselier L, Marshall BD, Reed J. 2000. Ecological benefits of riparian reforestation in urban watersheds: study design and preliminary results. Environmental Monitoring Assessment 63: 211-222. DOI: https://doi. org/10.1023/A:1006495805300

Hu W, Wang Y, Dong P, Zhang D, Yu W, Ma Z, Chen G, Liu Z, Du J, Chen B, Lei G. 2020. Predicting potential mangrove distribution at the global northern distribution margin using an ecological niche model: Determining conservation and reforestation involvement. Forest Ecology and Management 478: 1-13. DOI: https://doi.org/10.1016/j.foreco.2020.118517

INEGI [Instituto Nacional de Estadística y Geografía]. 2013. Modelo digital de elevación de alta resolución LIDAR, tipo terreno, escala 1:250,000. Aguascalientes, Aguascalientes. México. https://www.inegi.org.mx/ app/geo2/elevacionesmex/ (accessed May 13, 2020)

INEGI. 2016. Capa vectorial de uso de suelo y vegetación serie VI. Aguascalientes, Aguascalientes, México. http://www.conabio.gob.mx/informacion/gis/ (accessed March 4, 2020)

IPCC [Intergovernmental Panel on Climate Change]. 2013. Climate Change 2013: The Physical Science Basis. Contribution of Working Group I to the Fifth Assessment Report of the Intergovernmental Panel on Climate Change. United Kingdom-New York, USA: Cambridge University Press. 1535 pp. DOI: https://doi. org/10.1017/CBO9781107415324

Jayasinghe SL, Kumar L. 2019. Modeling the climate suitability of tea Camellia sinensis (L.) O. Kuntze in Sri Lanka in response to current and future climate change scenarios. Agricultural and Forest Meteorology 272-273: 102-117. DOI: https://doi.org/10.1016/j. agrformet.2019.03.025

Lobo JM, Jiménez-Valverde A, Real R. 2007. AUC: a misleading measure of the performance of predictive distribution models. Global Ecology and Biogeography 17: 145-151. DOI: https://doi.org/10.1111/j.1466$\underline{\text { 8238.2007.00358.x }}$

Manzanilla-Quijada GE, Treviño-Garza E, Vargas-Larreta B, López-Martínez JO, Mata-Balderas JM. 2020a. Áreas idóneas con potencial para la producción de semillas de Pinus chihuahuana Engelm. y Pinus leiophylla Scltdl. \& Cham. En México. Botanical Sci- ences 98: 305-316. DOI: https://doi.org/10.17129/ botsci. 2514

Manzanilla-Quijada GE, Treviño-Garza EJ, AguirreCalderón OA, Yerena-Yamallel JI, Manzanilla-Quiñones U. 2020b. Current and future potential distribution and identification of suitable areas for the conservation of Cedrela odorata L. in the Yucatan Peninsula. Revista Chapingo Serie Ciencias Forestales y del Ambiente 26: 163-180. DOI: https://doi.org/10.5154/r.rchscfa.2019.10.075

Manzanilla-Quiñones U, Aguirre-Calderón OA, JiménezPérez J, Treviño-Garza EJ, Yerena-Yamallel JI. 2019a. Distribución actual y futura del bosque subalpino de Pinus hartwegii Lindl en el Eje Neovolcánico Transversal. Madera y Bosques 25: 1-16. DOI: https://doi. org/10.21829/myb.2019.2521804

Manzanilla-Quiñones U, Delgado-Valerio P, HernándezRamos J, Molina-Sánchez A, García-Magaña JJ, Rocha Granados M de C. 2019b. Similaridad del nicho ecológico de Pinus montezumae y P. pseudostrobus (Pinaceae) en México: implicaciones para la selección de áreas productoras de semillas y de conservación. Acta Botánica Mexicana 126: 1-25.

Martin GM, Bellouin N, Collins WJ, Culverwell ID, Halloran PR, Hardiman SC, Hinton TJ, Jones CD, McDonald RE, McLaren AJ, O'Connor FM, Roberts MJ, Rodriguez JM, Woodward S, Best MJ, Brooks ME, Brown AR, Butchart N, Dearden C, Derbyshire SH, Dharssi I, Doutriaux-Boucher M, Edwards JM, Falloon PD, Gedney N, Gray LJ, Hewitt HT, Hobson M, Huddleston MR, Hughes J, Ineson S, Ingram WJ, James PM, Johns TC, Johnson CE, Jones A, Jones CP, Joshi MM, Keen AB, Liddicoat S, Lock AP, Maidens AV, Manners JC, Milton SF, Rae GL, Ridley JK, Sellar A, Senior CA, Totterdell IJ, Verhoef A, Vidale PL, Wiltshire A. 2011. The HadGEM2 family of Met Office Unified Model climate configurations. Geoscientific Model Development 4: 723-757. DOI: https://doi.org/10.5194/gmd4-723-2011

Martínez M. 1963. Las pináceas mexicanas, tercera edición. México, D.F: Instituto de Biología, Universidad Nacional Autónoma de México.

Martínez-Méndez N, Aguirre-Planter E, Eguiarte LE, Jaramillo-Correa JP. 2016. Modelado de nicho ecológico de las especies del género Abies (Pinaceae) en México: Algunas implicaciones taxonómicas y para la conservación. Botanical Sciences 94: 5-24. DOI: https://doi. org/10.17129/botsci.508

Martínez-Sifuentes AR, Villanueva-Díaz J, Manzanilla- 
Quiñones U, Becerra-López JL, Hernández-Herrera JA, Estrada-Ávalos J, Velázquez-Pérez A. 2020. Spatial modeling of the ecological niche of Pinus greggii Engelm. (Pinaceae): a species conservation proposal in Mexico under climate change scenarios. iForest Biogeosciences and Forestry 13: 426-434. DOI: https:// doi.org/10.3832/ifor3491-013

Medhaug I, Stolpe MB, Fischer EM, Knutti R. 2017. Reconciling controversies about the "global warming hiatus". Nature 545: 41-47. DOI: https://doi.org/10.1038/ nature 22315

Merow C, Smith MJ, Silander JA. 2013. A practical guide to MaxEnt for modeling species' distributions: what it does, and why inputs and settings matter. Ecography 36: 1058-1069. DOI: https://doi.org/10.1111/j.16000587.2013.07872.x

MEXU [Herbario Nacional de México] 2019. Universidad Nacional Autónoma de México. http://www.ib.unam. $\underline{\mathrm{mx} / \text { botanica/herbario. (accessed May 16, 2020) }}$

Monterrubio-Rico TC, Charre-Medellín JF, PachecoFigueroa C, Arriaga-Weiss S, Valdez-Leal JD, Cancino-Murillo R, Escalona-Segura G, Bonilla-Ruíz C, Rubio-Rocha Y. 2016. Distribución potencial histórica y contemporánea de la familia Psittacidae en México. Revista Mexicana de Biodiversidad 87: 1103-1117. DOI: http://dx.doi.org/10.1016/j.rmb.2016.06.004

Morrone JJ, Escalante T. 2016. Introducción a la biogeografía. México, DF: Universidad Nacional Autónoma de México. ISBN: 968-36-9463-2

Morton-Bermea O, Beramendi-Orosco L, MartínezReyes A, Hernández-Álvarez E, González-Hernández G. 2016. Increase in platinum group elements in Mexico City as revealed from growth rings of Taxodium mucronatum Ten. Environmental Geochemistry and Health 38: 195-202. DOI: https://doi.org/10.1007/ s10653-015-9703-2

Muscarella R, Galante PJ, Soley-Guardia M, Boria RA, Kass JM, Uriarte M. Anderson RP. 2014. ENMeval: An $R$ package for conducting spatially independent evaluations and estimating optimal model complexity for Maxent ecological niche models. Methods in Ecology and Evolutions 5: 1198-1205. DOI: https://doi. org/10.1111/2041-210X.12261

Naiman RJ, Decamps H, Pollock M. 1993. The role of riparian corridors in maintaining regional biodiversity. Ecological Applications 3: 209-212. DOI: https://doi. org/10.2307/1941822

Osorio-Olvera L, Vijay B, Narayani B, Soberón J, Falconi M. 2019. Ntbox: From getting biodiversity data to evaluating species distributions models in a friendly GUI environment. R package version 0.2.5.4. https:// github.com/luismurao/ntbox (accessed May 4, 2020)

Pérez-Miranda R, Romero-Sánchez ME, GonzálezHernández A, Rosales-Mata S, Moreno-Sánchez F, Arriola-Padilla VJ. 2019. Modelado de la distribución actual y bajo cambio climático de pinos piñoneros endémicos de México. Revista Mexicana de Ciencias Forestales 10: 218-237. DOI: https://doi.org/10.29298/ rmcf.v10i56.613

Peterson AT, Nakazawa Y. 2008. Environmental data sets matter in ecological niche modelling: an example with Solenopsis invicta and Solenopsis richteri. Global Ecology and Biogeography 17: 135-144. DOI: https:// doi.org/10.1111/j.1466-8238.2007.00347.x

Peterson AT. 2011. Ecological niche conservatism: a timestructured review of evidence. Journal of Biogeography 38: 817-827. DOI: https://doi.org/10.1111/j.13652699.2010.02456.x

Phillips SJ, Anderson RP, Schapire RE. 2006. Maximum entropy modeling of species geographic distributions. Ecological Modelling 190: 231-259. DOI: https://doi. org/10.1016/j.ecolmodel.2005.03.026

Phillips SJ, Dudik M. 2008. Modeling of species distributions with Maxent: new extensions and a comprehensive evaluation. Ecography 31: 161-175. DOI: https:// doi.org/10.1111/j.0906-7590.2008.5203.x

Pinedo-Alvarez C, Rentería-Villalobos M, Aguilar-Soto V, Vega-Mares JH, Melgoza-Castillo A. 2019. Distribution dynamics of Picea chihuahuana Martínez populations under different climate change scenarios in Mexico. Global Ecology and Conservation 17: e00559. DOI: https://doi.org/10.1016/j.gecco.2019. $\underline{\mathrm{e} 00559}$

R Core Team. 2015. $R$ : A language and environment for statistical computing. $R$ Foundation for Statistical Computing, Vienna, Austria. http://www.R-project.org/ (accessed April 12, 2020)

REMIB [Red Mundial de la Información sobre Biodiversidad]. 2019. Red Mundial de Información sobre Biodiversidad. México, DF: Comisión Nacional para el Conocimiento y Uso de la Biodiversidad. http://www. conabio.gob.mx/remib/doctos/remibnodosdb.html (accessed March 15, 2020)

Rzedowski J. 1986. Vegetación de México. Editorial Limusa. $432 \mathrm{pp}$

Sáenz-Romero C, Rehfeldt GE, Ortega-Rodríguez JM, Marín-Togo MC, Madrigal-Sánchez X. 2015. Pinus leiophylla Suitable Habitat For 1961-1990 and Future 
Climate. Botanical Sciences 93: 709-718. DOI: https:// doi.org/10.17129/botsci.86

SEMARNAT-CONANP [Secretaría de Medio Ambiente y Recursos Naturales-Comisión Nacional de Áreas Protegidas]. 2017. Archivo vectorial de Áreas Naturales Protegidas. Aguascalientes, Aguascalientes, México. http://www.conabio.gob.mx/informacion/gis/ (accessed April 5, 2020)

Soberón J, Townsend Peterson A. 2005. Interpretation of models of fundamental ecological niches and species distributions areas. Biodiversity Information 2: 1-10. DOI: https://doi.org/10.17161/bi.v2i0.4

SoilGrids. 2020. A system for digital soil mapping based on global compilation of soil profile data and environmental layers. https://soilgrids.org/ (accessed March 4, 2020)

Stockwell RB, Townsend Peterson A. 2002. Effects of sample size on accuracy of species distribution models. Ecological Modelling 148: 1-13. DOI: https://doi. org/10.1016/S0304-3800(01)00388-X

Suzán-Aspiri H, Enriquez-Peña G, Malda-Barrera G. 2007. Population structure of the Mexican baldcypress (Taxodium mucronatum Ten.) in Queretaro, Mexico. Forest Ecology and Management 242: 243-249. DOI: https://doi.org/10.1016/j.foreco.2007.01.041

Tiner RW. 1999. Wetland indicators: a guide to wetland, identification, delineation, classification and mapping. London, New York, Washington Lewis Publishers. Boca Raton: CRC press, 630 pp. ISBN: 978-143-985369-6

Vargas-Piedra G, Valdez-Cepeda RD, López-Santos A, Flores-Hernández A, Hernández-Quiroz NS, Martínez-Salvador M. 2020. Current and future potential distribution of the xerophytic scrub Candelilla ( $E u$ phorbia antisyphilitica) under two climate change scenarios. Forest 11: 2-15. DOI: https://doi.org/10.3390/ $\underline{\mathrm{f} 11050530}$

Villanueva Díaz J, Hernández Reyna A, García Sánchez F, Cornejo Oviedo E, Stahle DW, Therrell MD, Cleaveland MK. 2003. Análisis estructural de un rodal de sabino (Taxodium mucronatum Ten.) y vegetación circunvecina en los Peroles, San Luis Potosí, México. Ciencia Forestal 28: 57-79.

Villanueva Díaz J, Stahle D, Luckman BH, Cerano Pare- des J, Therrell MD, Morán Martínez R, Cleaveland MK. 2007. Potencial dendrocronológico de Taxodium mucronatum Ten. Y acciones para su conservación en México. Revista Ciencia Forestal en México 32: 9-37.

Villanueva-Díaz J, Cerano-Paredes J, Stahle DW, Constante-García V, Vázquez-Selem L, Estrada-Ávalos J, Benavides-Solorio JD. 2010. Árboles longevos de México. Revista Mexicana de Ciencias Forestales 1: $1-23$.

Villanueva-Díaz J, Constante-García V, Cerano-Paredes J, Martínez-Sifuentes AR, Stahle DW, Estrada-Ávalos J. 2013. Fenología y crecimiento radial del sabino (Taxodium mucronatum Ten.) en el río San Pedro Mezquital, Durango. Gómez Palacio, Durango, México: Centro Nacional de Investigaciones Forestales, Agrícolas y Pecuarias. Folleto técnico 27. ISBN: 978-607-370004-3

Villanueva-Díaz J, Constante-García V, Cerano-Paredes J, Martínez-Sifuentes AR. 2014. La cuenca San Martín y situación del ahuehuete (Taxodium mucronatum Ten.) en el río Sabinas, Coahuila. Gómez Palacio, Durango, México: Centro Nacional de Investigaciones Forestales, Agrícolas y Pecuarias. Folleto técnico 28. ISBN: 978-607-0319-2

Villanueva-Díaz J, Stahle DW, Therrell MD, BeramendiOrosco L, Estrada-Ávalos J, Martínez-Sifuentes AR, Astudillo-Sánchez CC, Cervantes-Martínez R, CeranoParedes J. 2020. The climatic response of baldcypress (Taxodium mucronatum Ten.) in San Luis Potosi, Mexico. Trees 34: 623-635. DOI: https://doi.org/10.1007/ s00468-019-01944-0

Villaseñor JL. 2016. Checklist of the native vascular plants of Mexico. Revista Mexicana de Biodiversidad 87: 559902. DOI: https://doi.org/10.1016/j.rmb.2016.06.017

Warren DL, Seifert SN. 2011 Ecological niche modeling in Maxent: The importance of model complexity and the performance of model selection criteria. Ecological Applications 21: 335-342. DOI: https://doi. org/10.1890/10-1171.1

Wu Y, Kuzma J, Marechal E, Graeff R, Lee HC, Foster R, Chua NH. 1997. Abscisic acid signaling through cucle ADP-ribose in plants. Sciences 278: 2126-2130. DOI: https://doi.org/10.1126/science.278.5346.2126
Editor de sección: Numa Pavón

Contribución de los autores: ARMS redacción, análisis espacial, elaboración de mapas. JVD estructura de escrito, revisión y redacción. ECR análisis espacial, obtención de registros y variables. DS revisión y estructura de estudio. 